\title{
How to Directly Measure Kondo Cloud's Length
}

\author{
Jinhong Park, ${ }^{1}$ S.-S. B. Lee, ${ }^{1}$ Yuval Oreg, ${ }^{2}$ and H.-S. Sim ${ }^{1, \text { * }}$ \\ ${ }^{1}$ Department of Physics, Korea Advanced Institute of Science and Technology, Daejeon 305-701, Korea \\ ${ }^{2}$ Department of Condensed Matter Physics, Weizmann Institute of Science, Rehovot 76100, Israel
}

(Dated: October 11, 2018)

\begin{abstract}
We propose a method to directly measure, by electrical means, the Kondo screening cloud formed by an Anderson impurity coupled to semi-infinite quantum wires, on which an electrostatic gate voltage is applied at distance $L$ from the impurity. We show that the Kondo cloud, and hence the Kondo temperature and the electron conductance through the impurity, are affected by the gate voltage, as $L$ decreases below the Kondo cloud length. Based on this behavior, the cloud length can be experimentally identified by changing $L$ with a keyboard type of gate voltages or tuning the coupling strength between the impurity and the wires.
\end{abstract}

PACS numbers: 72.15.Qm,73.63.Kv,72.10.Fk

Introduction.- The Kondo effect is a central manybody problem of condensed matter physics [1, 2]. It involves a spin singlet, formed by the spin-spin interaction between a magnetic impurity and surrounding conduction electrons. Deeper understanding of the effect has been achieved by using a quantum dot, that hosts a magnetic impurity spin, under systematic control 3 [ 6$]$ ].

Although the Kondo effect is well known, its spatial features still remain to be addressed. The Kondo spin singlet is formed below the energy scale of Kondo temperature $T_{K}$. This implies that the singlet is spatially formed over a conduction-electron region of length scale $\xi_{K}=\hbar v_{F} /\left(k_{B} T_{K}\right)$; when $T_{K} \sim 1 \mathrm{~K}$ and the Fermi velocity $v_{F} \sim 10^{5}-10^{6} \mathrm{~m} / \mathrm{s}, \xi_{K} \sim 1 \mu \mathrm{m}$. The region is called the Kondo screening cloud. There have been several proposals [7-19] for ways to detect the cloud.

Despite the proposals, there has been no conclusive measurement supporting the existence of the Kondo cloud 22, 23]. The difficulty to detect the cloud arises because it is a spin cloud showing quantum fluctuations with zero averaged spin. The cloud manifests itself in the spin-spin correlation [7-10] between the impurity and the conduction electrons. However it requires measurements of spin dynamics of time scale $\hbar /\left(k_{B} T_{K}\right)$. STM studies probing local density of states may be useful for detecting the cloud [12 14, 24]. Recent STM measurements 25 28] show the Kondo effect in the region away from a magnetic impurity, whose spatial extension is however much shorter than $\xi_{K}$. Another direction is to study a magnetic impurity in a finite-size system [15 21]. Because the cloud cannot extend beyond the finite size, the Kondo effect is strongly affected, and suppressed when the system is shorter than $\xi_{K}$. There has been no conclusive experimental detection of $\xi_{K}$ in this direction [21].

In this work, we propose a new way of detecting the Kondo cloud, based on the intuition that a change of conduction electrons inside the cloud will affect the Kondo effect. We consider a Kondo impurity formed in a quantum dot coupled to two semi-infinite ballistic quantum wires with electron tunneling amplitude $t_{W D}$ (see Fig.11).
Electrostatic gate voltages $V_{g}$ are applied to the wires (or to only one wire) at distance $L$ from the dot, modifying indirectly the local density of states $\rho(\epsilon)$ of conduction electrons nearby the dot (Fig. 2). We find that $V_{g}$ does not affect the cloud, when $L \gg \xi_{K}$. However, when $L \ll \xi_{K}$, the cloud, hence Kondo temperature $T_{K}$ and electron conductance $G$ through the dot, are sensitive to $V_{g}$. The crossover between the two regimes occurs at $L \approx \xi_{K}$. By measuring $G$ or $T_{K}$ with varying $L$ or $t_{W D}$ (Figs. 3 and 4), one can detect the crossover and $\xi_{K}$. We use the poor man scaling 29], numerical renormalization group study (NRG) 30 32], and Fermi liquid theory [33].

The setup.- We describe the wires by the tightbinding Hamiltonian with sites $j$ 's in wire $i=l, r$,

$$
\begin{aligned}
H_{W}= & \sum_{i=l, r} \sum_{j=1}^{\infty} \sum_{\sigma=\uparrow, \downarrow}\left[\epsilon_{0} n_{i j \sigma}+\left(-t c_{i j \sigma}^{\dagger} c_{i(j+1) \sigma}+\text { H.c. }\right)\right] \\
& -e V_{g} \sum_{i=l, r} \sum_{j=N+1}^{\infty} \sum_{\sigma=\uparrow, \downarrow} n_{i j \sigma}
\end{aligned}
$$

where $c_{i j \sigma}^{\dagger}$ creates an electron with spin $\sigma$ and energy $\epsilon_{0}$ at site $j$ in wire $i, n_{i j \sigma} \equiv c_{i j \sigma}^{\dagger} c_{i j \sigma}$, and $t$ is the hopping energy. The last term describes the gate voltage $V_{g}$ applied in $|x|>L=N a$, where $a$ is the lat-

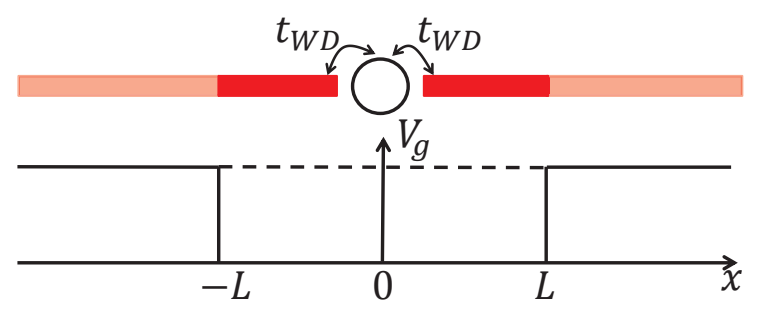

FIG. 1: (Color Online) Setup for detecting the Kondo cloud. A quantum dot located at $x=0$ hosts a magnetic impurity spin. It couples to quantum wires along $\hat{x}$ axis, with electron tunneling amplitude $t_{W D}$. Gate voltage $V_{g}$ is applied at distance $L$ from the dot (in $|x|>L$ ). The Kondo effect becomes sensitive to $V_{g}$, as $L$ decreases below the cloud length. 
tice spacing. $V_{g}(x)$ changes at $x=L$ abruptly over the length shorter than the Fermi wave length. The dot Hamiltonian $H_{D}$ is modeled by the Anderson impurity [34], $H_{D}=\sum_{\sigma=\uparrow, \downarrow} \epsilon_{d} d_{\sigma}^{\dagger} d_{\sigma}+U n_{d \uparrow} n_{d \downarrow}$, where $d_{\sigma}^{\dagger}$ creates an electron with energy $\epsilon_{d}$ and spin $\sigma$ in the dot, $n_{d \sigma} \equiv d_{\sigma}^{\dagger} d_{\sigma}$, and $U$ is the electron repulsive interaction. $H_{T}=-t_{W D} \sum_{i} \sum_{\sigma=\uparrow, \downarrow}\left(c_{i 1 \sigma}^{\dagger} d_{\sigma}+\right.$ H.c. $)$ describes electron tunneling between the wire and the dot.

The dot is occupied by a single electron in the Coulomb blockade regime of $\epsilon_{d}<\epsilon_{F}$ and $\Gamma\left(\epsilon_{F}\right) \ll-\epsilon_{d}+\epsilon_{F}, U+$ $\epsilon_{d}-\epsilon_{F}$, where $\Gamma(\epsilon)=2 \pi\left|t_{W D}\right|^{2} \rho(\epsilon)$ is the hybridization function between the dot and the wires, $\rho(\epsilon)$ is the local density of states at energy $\epsilon$ in the neighboring sites $j=1$ of the dot, and $\epsilon_{F}$ is the Fermi energy. In this regime, the total Hamiltonian of the setup becomes [2, 6, 35]

$$
H=H_{D}+H_{W}+H_{T} \simeq J \vec{s} \cdot \vec{S}+V \sum_{i, \sigma} n_{i 1 \sigma}+H_{W} .
$$

Here, the Kondo impurity spin, $\vec{S}=\sum_{\sigma, \sigma^{\prime}} d_{\sigma}^{\dagger} \vec{\sigma}_{\sigma \sigma^{\prime}} d_{\sigma^{\prime}} / 2$, couples with the spin of the neighboring conduction electrons, $\vec{s}=\sum_{\sigma, \sigma^{\prime}}\left(c_{l 1 \sigma}^{\dagger}+c_{r 1 \sigma}^{\dagger}\right) \vec{\sigma}_{\sigma \sigma^{\prime}}\left(c_{l 1 \sigma^{\prime}}+c_{r 1 \sigma^{\prime}}\right) / 2$, with strength $J=2 t_{W D}^{2}\left[-1 /\left(\epsilon_{d}-\epsilon_{F}\right)+1 /\left(U+\epsilon_{d}-\epsilon_{F}\right)\right]$. The second term describes the potential scattering with strength $V=t_{W D}^{2}\left[-1 /\left(\epsilon_{d}-\epsilon_{F}\right)-1 /\left(U+\epsilon_{d}-\epsilon_{F}\right)\right] / 2$.

Local density of states. - We show how the gate voltage $V_{g}$ changes the local density of states $\rho(\epsilon)$ at sites $j=1$ of the wires. We calculate $\rho(\epsilon)$, by matching the singleparticle wavefunctions of $H_{W}$ between $j=N$ and $N+1$,

$$
\rho(\epsilon)=\frac{\sin (q a) \sin ^{2}(k a)}{\pi a t\left[\sin ^{2}(k a)+\frac{e V_{g}}{t} \sin [k(N+1) a] \sin (k N a)\right]} .
$$

$k$ and $q$ are the wavevectors in $|x|<L$ and $|x|>L$, respectively, satisfying $\epsilon=\epsilon_{0}-2 t \cos (k a)=\epsilon_{0}-e V_{g}-$ $2 t \cos (q a) . \quad \Gamma(\epsilon)=2 \pi t_{W D}^{2} \rho(\epsilon)$ shows resonances with level spacing $\Delta \equiv \pi \hbar v_{F} / L$; see Fig. 2 The oscillation amplitude of $\Gamma(\epsilon)$ is proportional to $V_{g}$. The value of $\Gamma\left(\epsilon_{F}\right)$ of the $L \rightarrow \infty$ limit is denoted as $\Gamma_{\infty}$, which equals the average of $\Gamma(\epsilon)$ around $\epsilon_{F}$ for finite $L$.

We sketch how the change of $\rho(\epsilon)$ by $V_{g}$ affects the Kondo effect in different regimes of $L$. For $L \gg \xi_{K \infty}$ (i.e., $\left.T_{K \infty} \gg \Delta\right)$, the Kondo temperature is determined by $\Gamma_{\infty}$ as $T_{K \infty} \sim \sqrt{\Gamma_{\infty} U / 2} \exp \left[\pi\left(\epsilon_{d}-\epsilon_{F}\right)\left(U+\epsilon_{d}-\epsilon_{F}\right) /\left(2 \Gamma_{\infty} U\right)\right]$, and the cloud size is $\xi_{K \infty}=\hbar v_{F} /\left(k_{B} T_{K \infty}\right)$. In this regime, the average $\Gamma_{\infty}$ of many resonances of $\Gamma(\epsilon)$ around $\epsilon_{F}$ determines the Kondo effect, insensitively to $V_{g}$. On the other hand, for $L \ll \xi_{K \infty}$ (i.e., $T_{K \infty} \ll \Delta$ ), the resonance of $\Gamma(\epsilon)$ located at $\epsilon_{F}$, namely $\Gamma_{0} \equiv \Gamma\left(\epsilon_{F}\right)$, determines the Kondo effect, resulting in $T_{K}=T_{K 0} \sim$ $\sqrt{\Gamma_{0} U / 2} \exp \left[\pi\left(\epsilon_{d}-\epsilon_{F}\right)\left(U+\epsilon_{d}-\epsilon_{F}\right) /\left(2 \Gamma_{0} U\right)\right]$ and $\xi_{K}=$ $\hbar v_{F} /\left(k_{B} T_{K 0}\right)$. In this case, $V_{g}$ affects conduction electrons within the cloud, and modifies $T_{K}$.

We will discuss how $T_{K}$ changes between $T_{K 0}$ and $T_{K \infty}$ as a function of $L / \xi_{K}$ in the two possible situations, case A where one changes $L$ with keeping $t_{W D}$ constant, and case $\mathrm{B}$ where $t_{W D}$ changes and $L$ remains constant.

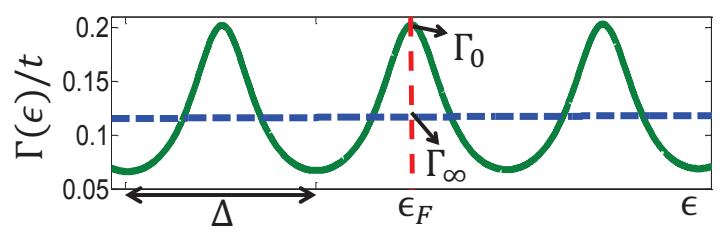

FIG. 2: (Color Online) Hybridization function $\Gamma(\epsilon)=$ $2 \pi t_{W D}^{2} \rho(\epsilon)$ in unit of $t$ for infinite (blue dashed curve) and finite $L$ (green solid); we choose $\epsilon_{0} / 2 t=0.931, t_{W D} / 2 t=0.2$, and $e V_{g} / 2 t=0.125$. For finite $L, \Gamma(\epsilon)$ has resonances with spacing $\Delta \equiv \pi \hbar v_{F} / L ; \epsilon_{F}$ is chosen to be located at a resonance center (red dashed line). When $L$ is so large that $V_{g}$ is applied outside the Kondo cloud, the Kondo temperature $T_{K}=T_{K \infty}$ is determined by $\Gamma_{\infty}$ and independent of $V_{g}$. On the other hand, $T_{K}$ depends on $V_{g}$ and $L$, when $L \lesssim$ the cloud size $\xi_{K}$. When $L \ll \xi_{K}, T_{K}$ is determined by $\Gamma_{0} \equiv \Gamma\left(\epsilon_{F}\right)$.

Kondo temperature.- We compute $T_{K}$, using the poor man scaling and the NRG 32].

In the poor man scaling, the renormalization of $J \rightarrow$ $J+J^{2}\left(\int_{-D_{0}}^{-D}+\int_{D}^{D_{0}}\right) d \epsilon \rho(\epsilon) /|\epsilon|$ is performed with reducing the energy bandwidth of the wire from $D_{0}$ to $D$, and stopped at the bandwidth where $J^{2} \int d \epsilon \rho(\epsilon) /|\epsilon|$ is comparable with $J$. The final bandwidth provides $T_{K}$,

$$
\begin{aligned}
\ln \left(\frac{T_{K}}{T_{K \infty}}\right) & \simeq-\frac{e V_{g} \cos \left(k_{F}(2 L+a)\right)}{2 t \sin ^{2}\left(k_{F} a\right)} \operatorname{Ci}\left(\frac{2 L}{\xi_{K}}\right) \\
& \simeq-\frac{e V_{g}}{2 t \sin ^{2}\left(k_{F} a\right)} \operatorname{Ci}\left(\frac{2 L}{\xi_{K}}\right) \text { for } k_{F}=k_{F, n}
\end{aligned}
$$

where $\operatorname{Ci}(y) \equiv \int_{-\infty}^{-y} d y^{\prime}\left(\cos y^{\prime}\right) / y^{\prime}, k_{F, n}=2 \pi n /(2 L+a)$, and $n$ is an integer. Equation (4) is obtained by putting $k \rightarrow k_{F} \equiv k\left(\epsilon_{F}\right), q \rightarrow q\left(\epsilon_{F}\right), L \gg a,\left.v_{F} \equiv \frac{1}{\hbar} \frac{\partial \epsilon}{\partial k}\right|_{k_{F}}=$ $(2 a t / \hbar) \sin \left(k_{F} a\right),\left|e V_{g}\right| \ll 2 t \sin ^{2}\left(k_{F} a\right)$, and the linearization of $\epsilon \simeq \epsilon_{F}+\hbar v_{F}\left(k-k_{F}\right)$ into Eq. (3); $k \rightarrow k_{F}$ and $q \rightarrow q\left(\epsilon_{F}\right)$ are valid within the small energy scale of $T_{K}$. We remark that $\xi_{K}$ depends on $L$ and $V_{g}$ in Eq. (4).

In Eq. (4), the term $\operatorname{Ci}\left(2 L / \xi_{K}\right)$ gives the information on the cloud, while another $L$ dependence of the $2 k_{F}$ oscillation appears because resonance centers in $\Gamma(\epsilon)$ shift across $\epsilon_{F}$ as $L$ changes. One can focus on the former. In case $\mathrm{A}$, where one changes $L$, one can reduce the effect of the $2 k_{F}$ oscillation, by considering the situation that $\epsilon_{F}$ is located near the bottom of an energy band where the $2 k_{F}$ term slowly oscillates, or by considering the resonance condition of $k_{F}=k_{F, n}$ where the $2 k_{F}$ term provides the maximum value; see Eq. (5). The resonance condition can be achieved at each value of $L$, by tuning an additional gate voltage applied to the entire region of the wires (not shown in Fig. 11) with monitoring the conductance through the dot. On the other hand, in case B, where one changes $t_{W D}$ with keeping $L$ constant, the term $\cos \left(k_{F}(2 L+a)\right)$ is constant, hence can be ignored.

For case A under the resonance situation of $k_{F}=k_{F, n}$, the poor man scaling in Eq. (5) is plotted as a function of $L / \xi_{K \infty}$ (rather than $L / \xi_{K}$ ) in Fig. B(a). As expected, $T_{K}$ 

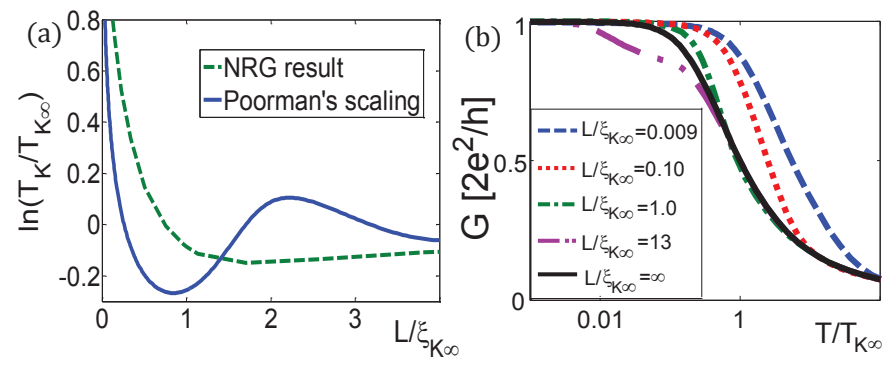

FIG. 3: (Color Online) Case A under the resonance condition of $k_{F}=k_{F, n}$. In this case, one changes $L$ with keeping $t_{W D}$ (hence $T_{K \infty}$ and $\xi_{K \infty}$ ) constant. (a) Kondo temperature $T_{K}$ as a function of $L$, obtained by the poor man scaling (blue solid curve) and NRG (green dashed). The two approaches show qualitatively the same overall behavior that $T_{K}$ drastically changes for $L \lesssim \xi_{K \infty}$, while $T_{K} \sim T_{K \infty}$ for $L \gtrsim \xi_{K \infty}$; their discrepancy in $L \gtrsim \xi_{K \infty}$ is discussed in the text. (b) NRG result of the temperature $T$ dependence of conductance $G$ for various values of $L / \xi_{K \infty}$. We choose $\Gamma_{\infty} / 2 t=0.28$, $\epsilon_{0} / 2 t=0.925, e V_{g} / 2 t=0.125$, and $U / 2 t=3.6$.

stays at $T_{K \infty}$ for $\xi_{K \infty} \lesssim L$, drastically changes around $L=\xi_{K \infty}$, and approaches to $T_{K 0}$ for $L \ll \xi_{K}$. In addition, the oscillation of $\ln \left(T_{K} / T_{K \infty}\right) \sim \operatorname{sinc}\left(2 L / \xi_{K \infty}\right)$ appears for $L \gtrsim \xi_{K \infty}$; we put $\mathrm{Ci}(x) \sim \operatorname{sinc} x=(\sin x) / x$ and $\xi_{K} \simeq \xi_{K \infty}$ (valid for $x=2 L / \xi_{K \infty} \gtrsim 1$ ) into Eq. (4). The oscillation originates from the average effect of $\rho(\epsilon)$ within $T_{K \infty}$, and becomes suppressed for longer $L$ as more $\left(\sim T_{K \infty} / \Delta\right)$ resonances appear within $T_{K \infty}$. On the other hand, for $L \ll \xi_{K \infty}$, we find $\ln \left(T_{K} / T_{K \infty}\right) \propto$ $-\ln \left(L / \xi_{K}\right)$, using $\operatorname{Ci}(x) \sim \ln (x)+0.577$ for $x \ll 1$. The above behavior of $T_{K}\left(L / \xi_{K \infty}\right)$ reveals the Kondo cloud.

Conductance.- We compute the temperature $T$ dependence of electron conductance $G$ between the wires through the dot, using the NRG [17, 30 32]. We will discuss how to extract $\xi_{K \infty}$ from $G(T)$ in cases A and B.

We continue to discuss case A under the resonance condition of $k_{F}=k_{F, n}$. In Fig. [3(b), we plot $G(T)$ for different $L$ 's. It is custom [36] to get an estimate for $T_{K}$ from the temperature at which $G(T)$ equals the half of the zero-temperature conductance $G(T=0, L \rightarrow \infty)$ of the $L \rightarrow \infty$ case. $G(T)$ shows the behavior distinct between $L \gtrsim \xi_{K \infty}$ and $L \lesssim \xi_{K \infty}$. For $L \gtrsim \xi_{K \infty}, G(T)$ equals $G(T=0, L \rightarrow \infty) / 2$ at almost the same temperature, implying that $T_{K}$ equals $T_{K \infty}$ independent of $L$. On the other hand, for $L \lesssim \xi_{K \infty}, G(T)$ shows that $T_{K}$ changes toward $T_{K 0}$ as $L$ decreases. This NRG result agrees with the poorman scaling; see Fig. 3(a). In this way, one directly measures $\xi_{K \infty}$.

There is a discrepancy between the two curves in Fig. 3(a). In the NRG case, $\ln \left(T_{K} / T_{K \infty}\right)$ decreases only monotonously for $L \gtrsim \xi_{K}$, without showing the behavior $\operatorname{sinc}\left(2 L / \xi_{K \infty}\right)$ of the poor man scaling. The discrepancy may come from the known limitation that the logarithmic discretization scheme of NRG does not perfectly capture the behavior of high-energy states (higher than $\Delta$
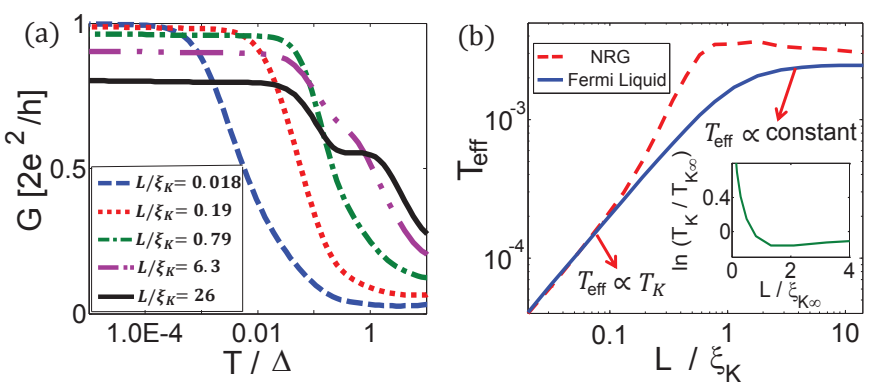

FIG. 4: (Color Online) Case B. In this case, one changes $t_{W D}$ (hence $\xi_{K}$ ) with keeping $L$ constant. (a) The NRG result of $G(T)$ for different values of $t_{W D}$; we show the values of $\xi_{K}$ instead of $t_{W D}$. (b) $T_{\text {eff }}\left(L / \xi_{K}\right)$ [defined in Eq. (8)], obtained from the two different approaches of the Fermi liquid theory and the NRG. Inset: The NRG result of $T_{K}\left(L / \xi_{K \infty}\right)$ exhibits the same behavior as Fig. 33(a). We choose $L=100 a, \epsilon_{0} / 2 t=$ $0.931, e V_{g} / 2 t=0.125$, and $U / 2 t=3.6$. $\epsilon_{F}$ is chosen to be located at a center of a resonance of $\Gamma(\epsilon)$, for simplicity.

in our case). On the other hand, for $L \ll \xi_{K \infty}$, where low-energy states mainly contribute to the Kondo effect, the NRG shows the same behavior of $\ln \left(T_{K} / T_{K \infty}\right) \sim$ $-\ln \left(L / \xi_{K}\right)$ as the poorman scaling.

Next, we discuss case B where one changes $t_{W D}$ with keeping $L$ constant (hence $\Delta=\pi \hbar v_{F} / L$ is constant). Figure 4 shows the NRG result of $G(T)$ for different $\xi_{K}$ 's. We obtain $T_{K}$ from the high-temperature behavior of $G(T)$ in the same way as above, by choosing the temperature at which $G(T)=G(T=0, L \rightarrow \infty) / 2$. The result of $T_{K}$ agrees with case A; see the inset of Fig. प(b). We below suggest another way to see the cloud from the lowtemperature behavior of $G(T)$. Note that as $T$ changes across $\Delta, G(T)$ can show a jump due to the resonance structure of $\rho(\epsilon)$, as shown for $L / \xi_{K}=26$ in Fig. 4(a).

We describe the regime of $T \ll T_{K}, \Delta$, using the fixedpoint Hamiltonian of the Fermi liquid theory [6, 33, 37],

$$
\begin{aligned}
H_{\mathrm{low}} \simeq & \sum_{k \sigma} \epsilon_{k} c_{k \sigma}^{\dagger} c_{k \sigma}-\frac{1}{\pi \rho\left(\epsilon_{F}\right)} \sum_{k k^{\prime} \sigma}\left(\frac{\epsilon_{k}+\epsilon_{k^{\prime}}}{2 T_{K}}+\delta_{p}\right) c_{k \sigma}^{\dagger} c_{k^{\prime} \sigma} \\
& +\frac{1}{\pi T_{K} \rho^{2}\left(\epsilon_{F}\right)} \sum_{k_{1} k_{2} k_{3} k_{4}} c_{k_{1} \uparrow}^{\dagger} c_{k_{2} \uparrow} c_{k_{3} \downarrow}^{\dagger} c_{k_{4} \downarrow}
\end{aligned}
$$

where $c_{k \sigma}^{\dagger}$ creates an electron with momentum $k$, spin $\sigma$, and energy $\epsilon_{k} . \delta_{p}$ is the phase shift by the potential scattering, which occurs as the particle-hole symmetry is broken. Although $\rho$ depends on $\epsilon$, we take for simplicity $\rho\left(\epsilon_{F}\right)$ in Eq. (6) as a crude approximation. The second term of Eq. (6) describes elastic scattering of electrons by the Kondo singlet, with scattering phase shift $\delta(\epsilon)=\pi / 2+\left(\epsilon-\epsilon_{F}\right) / T_{K}+\delta_{p}$. The third term shows repulsive interactions that break the Kondo singlet, and contributes to inelastic T-matrix $t^{\text {in }}$ as $-\pi \rho\left(\epsilon_{F}\right) \operatorname{Im} t^{\text {in }}(\epsilon)=\left[\left(\epsilon-\epsilon_{F}\right)^{2}+\pi^{2} T^{2}\right] /\left(2 T_{K}^{2}\right)$. By com- 
bining $\delta(\epsilon)$ and $\operatorname{Im} t^{\mathrm{in}}(\epsilon)$, we obtain [6]

$$
\begin{aligned}
G(T) & =\frac{2 e^{2}}{h} \frac{1+\cos \left(2 \delta_{p}\right)}{2}\left(1-\frac{\pi^{2} T^{2}}{T_{\mathrm{eff}}^{2}}\right), \\
\frac{1}{T_{\mathrm{eff}}^{2}} & \equiv \frac{2 \beta}{3(\alpha+\beta) \Delta^{2}}+\frac{2 \cos \left(2 \delta_{p}\right)}{\left(1+\cos \left(2 \delta_{p}\right)\right) T_{K}^{2}},
\end{aligned}
$$

where $\alpha$ and $\beta$ are constants depending on $V_{g}$ and $k_{F}$ but independent of $L . \delta_{p}$ is obtained by comparing $G(T=$ $0)$ with Eq. (7). For nonzero $\delta_{p}, G(T=0)$ deviates from the unitary-limit value of $2 e^{2} / h$. Note that when $\rho$ is independent of $\epsilon$ and the particle-hole symmetry is preserved, $\delta_{p}=0$ and $\beta=0$, hence, $T_{\text {eff }} \rightarrow T_{K}$.

$T_{\text {eff }}$ is obtained by comparing $G(T)$ with Eq. (7) in experiments or in the NRG, while computed from Eq. (8) in the Fermi liquid theory. We plot $T_{\text {eff }}\left(L / \xi_{K}\right)$ in Fig. 4(b), showing good agreement between the NRG and the Fermi liquid theory; their quantitative discrepancy may come from our approximation in the Fermi liquid theory.

The dependence of $T_{\text {eff }}$ on $L / \xi_{K}$ or $L / \xi_{K \infty}$ is useful for identifying $\xi_{K}$ in case $\mathrm{B}$, since $\Delta$ is constant so that $T_{\text {eff }}\left(L / \xi_{K}\right)$ directly provides the information of $T_{K}\left(L / \xi_{K}\right)$; see Eq. (8). For $L \gtrsim \xi_{K}, T_{\text {eff }}$ is almost constant, implying that $T_{K}$ and $\xi_{K}$ are independent of $L$. For $L \lesssim \xi_{K}, T_{\text {eff }}$ (hence $T_{K}$ ) depends on $L / \xi_{K}$. The crossover occurs around $\xi_{K} \simeq L$.

Discussion.- Our proposal may be within experimental reach. Case A, where $L$ varies, may be achieved with keyboard-type gate voltages, while one tunes $t_{W D}$ by a gate in case B. A good candidate for our proposal may be a carbon nanotube, where $T_{K} \sim 1 \mathrm{~K}$ and $\xi_{K} \sim 1 \mu \mathrm{m} \mathrm{38}$.

For both the cases, a (single-mode or multi-mode) wire whose Fermi level $\epsilon_{F}$ lies near the bottom $E_{b}$ [van Hove singularity (VHS)] of one of the energy bands is useful to achieve a conclusive evidence of the cloud; our results of Eq. (4) and NRG are applicable to this regime, since they are obtained, taking into account of the energy dependence of $\rho$. In this regime, $\rho(\epsilon)$ is sensitive to $V_{g}$, hence, it may not be difficult to obtain sizable difference between $T_{K \infty}$ and $T_{K 0}$ by $V_{g}\left(<\epsilon_{F}-E_{b}\right)$. Our analysis for a single-mode wire is applicable, without modification, to a multi-mode wire, since the band near the VHS governs the Kondo effect dominantly over the other modes. Moreover, the Fermi wave length $k_{F}^{-1}$ of the band near the VHS can satisfy $k_{F}^{-1} \gg L, \xi_{K}, l_{s}$, where $l_{s}$ is the length scale over which $V_{g}(x)$ spatially changes from 0 to $V_{g}$ at $x=L$. Then, the $2 k_{F}$ term in Eq. (4) oscillates slowly as a function of $L$ in the range of $L$ where the transition between $T_{K \infty}$ and $T_{K 0}$ occurs; in the case of smooth gate potentials with $k_{F} l_{s} \gg 1$, the $2 k_{F}$ oscillations will be washed out. Finally, to avoid any effects of $V_{g}$ on $G$ irrelevant to the Kondo effect, one may consider a quantum dot coupled to three wires, applying $V_{g}$ to one of the wires and measuring $G$ between the other two.

Note that our system is distinct from the Kondo box (a Kondo impurity in a finite-size system) [17 21]. In the latter, the cloud is terminated hence strongly modified by the box boundary, hence, it is hard to directly detect $\xi_{K}$. In contrast, in our system, the cloud is extensible to $|x|>L$ (not suppressed even for $L \ll \xi_{K}$ ) and can be only weakly (perpurbatively) modified, allowing direct detection of $\xi_{K}$ and the spatial structure of the cloud.

We thank I. Affleck, G. Finkelstein, L. Glazman, D. Goldharber-Gordon, S. Ilani, A. K. Mitchell, S. Tarucha for useful discussions, and Minchul Lee for advices on NRG calculations. We acknowledge support by NRF (grant 2011-0022955; HSS), and by BSF and Minerva grants (YO). HSS thanks J. Moore and UC Berkeley, where this paper is written, for hospitality.

* Electronic address: hssim@kaist.ac.kr

[1] J. Kondo, Prog. Theor. Phys. 32, 37 (1964).

[2] A. C. Hewson, The Kondo Problem to Heavy Fermions (Cambridge University Press, Cambridge, 1993).

[3] D. Goldhaber-Gordon et al., Nature 391, 156 (1998).

[4] S. M. Cronenwett, T. H. Oosterkamp, and L. P. Kouwenhoven, Science 281, 540 (1998).

[5] L. Kouwenhoven and L. I. Glazman, Physics World 14, 33 (2001).

[6] L. I. Glazman and M. Pustilnik, in Nanophysics: Coherence and Transport, eds. H. Bouchiat et al. (Elsevier, 2005), pp. 427-478.

[7] J. E. Gubernatis, J. E. Hirsch, and D. J. Scalapino, Phys. Rev. B 35, 8478 (1987).

[8] V. Barzykin and I. Affleck, Phys. Rev. Lett. 76, 4959 (1996); Phys. Rev. B 57, 432 (1998).

[9] L. Borda, Phys. Rev. B 75, 041307 (2007).

[10] A. Holzner, I. P. McCulloch, U. Schollwöck, J. von Delft, and F. Heidrich-Meisner, Phys. Rev. B 80, 205114 (2009).

[11] E. S. S $\phi$ rensen and I. Affleck, Phys. Rev. B 53, 9153 (1996).

[12] I. Affleck, L. Borda, and H. Saleur, Phys. Rev. B 77, 180404 (2008).

[13] C. A. Büsser et al., Phys. Rev. B 81, 045111 (2010).

[14] A. K. Mitchell, M. Becker, and R. Bulla, Phys. Rev. B 84115120 (2011).

[15] I. Affleck and P. Simon, Phys. Rev. Lett. 86, 2854 (2001).

[16] R. Yoshii and M. Eto, Phys. Rev. B 83165310 (2011).

[17] P. S. Cornaglia and C. A. Balseiro, Phys. Rev. B 66, 115303 (2002); Phys, Rev. Lett. 90, 216801 (2003).

[18] P. Simon and I. Affleck, Phys. Rev. Lett. 89, 206602 (2002); Phys. Rev. B 68, 115304 (2003).

[19] T. Hand, J. Kroha, and H. Monien, Phys. Rev. Lett. 97, 136604 (2006).

[20] W. B. Thimm, J. Kroha, and J. von Delft, Phys. Rev. Lett. 82, 2143 (1999).

[21] Yu. Bomze et. al., Phys. Rev. B 82, 161411 (2010).

[22] For a review, see I. Affleck, in Perspectives of Mesoscopic Physics (World Scientific, 2010), pp. 1-44.

[23] J. P. Boyce and C. P. Slichter, Phys. Rev. Lett. 32, 61 (1974); Phys. Rev. B 13, 379 (1976).

[24] G. Bergmann, Phys. Rev. B 77, 104401 (2008).

[25] V. Madhavan et al., Science 280, 567 (1998).

[26] H. C. Manoharan, C. P. Lutz, and D. M. Eigler, Nature 
403, 512 (2000).

[27] H. Prüser et al., Nat. Phys. 7, 203 (2011).

[28] Y.-S. Fu et al., Phys. Rev. Lett. 99, 256601 (2007).

[29] P. W. Anderson, J. Phys. C 3, 2439 (1970).

[30] K. G. Wilson, Rev. Mod. Phys. 47, 773 (1975); H. R. Krishna-murthy, J. W. Wilkins, and K. G. Wilson, Phys. Rev. B 21, 1003 (1980).

[31] R. Bulla, T. A. Costi, and T. Pruschke, Rev. Mod. Phys. 80, 395 (2008).

[32] We use the full desnity matrix NRG method developed by A. Weichselbaum and J. von Delft, Phys. Rev. Lett. 99, 076402 (2007). We use the NRG discretization parameter of $\Lambda=2$ and keep $\sim 300$ states at each iteration.
[33] P. Nozières, J. Low Temp. Phys. 17, 31 (1974); J. Phys. (Paris) 39, 1117 (1978).

[34] P. W. Anderson, Phys. Rev. 124, 41 (1961).

[35] J. R. Schrieffer and P. A. Wolff, Phys. Rev. 149, 491 (1966).

[36] D. Goldhaber-Gordon et al., Phys. Rev. Lett. 81, 5225 (1998).

[37] I. Affleck and A. W. W. Ludwig, Phys. Rev. B 48, 7297 (1993).

[38] J. Nygard, D. H. Cobden, and P. E. Lindelof, Nature 408, 342 (2000). 